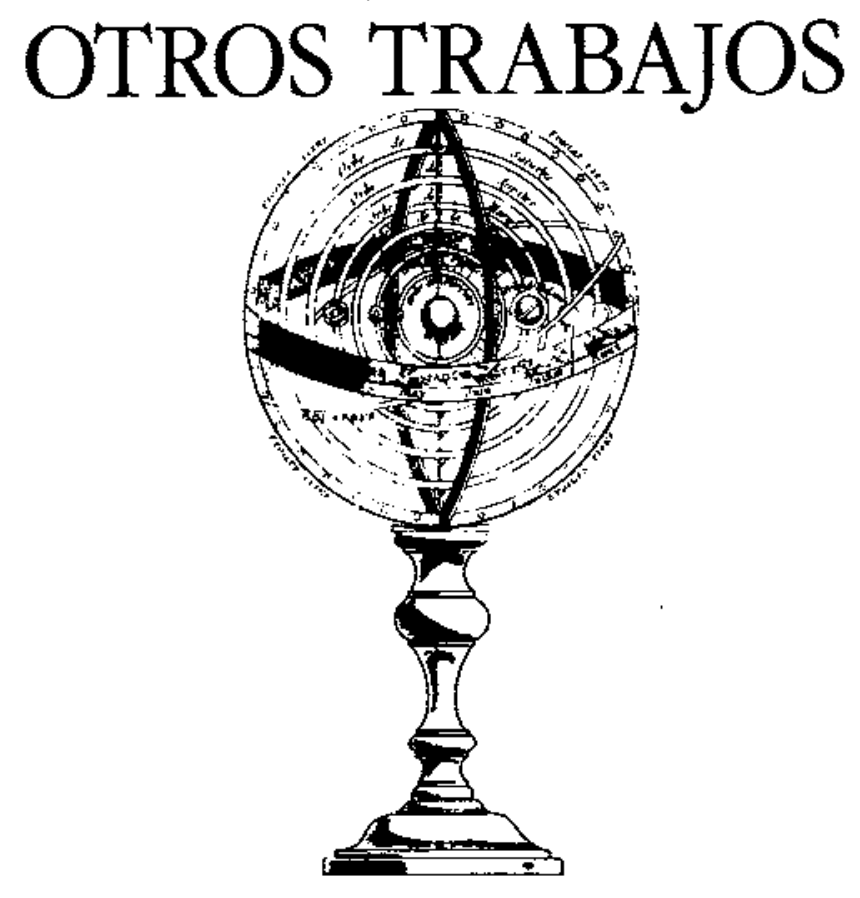

\title{
LA ELABORACIÓN DE PROYECTOS CURRICULARES DE CENTRO EN EL MARCO DE UN CURRICULO DE CIENCIAS ABIERTO
}

\author{
DEL CARMEN, $L$. \\ ICE de la Universidad de Barcelona.
}

Ponencia presentada al III Congreso Internacional sobre Didáctica de las Ciencias Experimentales y las Matemáticas, celebrado en Santiago de Compostela en Septiembre de 1989.

\section{SUMMARY}

The new curricular proposeds put foreword by the Ministry of Education are characterized, among other things, by being based on a flexible, open design, whose development requires active participation of the teaching teams in each school. The characteristics of this model are analysed as well as the necessary conditions for its viability with special reference to science teaching. The problem of organization and grading educational contents are emphesized.

\section{INTRODUCCIÓN}

De las nuevas propuestas curriculares presentadas a debate por el MEC (abril, 1989) resaltan, entre otras, dos características básicas:

* La adopción đe un diseño curricular abierto y flexible.

* Y en función de él, la necesidad de que los equipos de profesores de los centros educativos elaboren Proyec- tos Curriculares de Centro, que posibiliten la adecuación del Diseño Curricular Base propuesto por la Administración educativa, a las condiciones específicas de cada contexto particular.

El presente trabajo se plantea aportar algunos elementos para el análisis y la reflexión sobre ambos aspectos, procediendo para ello a comentar: 
* El sentido que se atribuye al término currículo abierto, en las propuestas ministeriales.

* El significado de los Proyectos Curriculares de Centro (PCC) en este contexto, y sus diferencias en relación a otras acepciones del término proyecto curricular.

* Y Ios elementos básicos que deben contemplarse en la elaboración del PCC, haciendo referencia al tema de la secuenciación de contenidos.

\section{EL GRADO DE APERTURA Y FLEXIBI- LIDAD DEL CURRICULO}

El actual Proyecto de Reforma Educativa(MEC, 1989), se plantea una distribución de competencias y responsabilidades en el proceso de elaboración, desarrolio y control del currículo, que otorga un protagonismo considerable a los equipos docentes de los centros educativos. Sólo así, se argumenta (Coll 1989), será posible llevar a cabo una educación que tenga en cuenta los diversos factores de cada situación educativa particular, respetuosa con la diversidad, y ajustada a los intereses, motivaciones y capacidades de los alumnos.

Este planteamiento, reivindicado durante muchos años por los movimientos de renovación pedagógica y los grupos de profesores más innovadores, parece bastante adecuado, ya que como señala Ángel Pérez (1986): "Si se admite el carácter singular de cada contexto escolar y del aula, el profesor no puede ser un mero técnico que aplique una secuencia de rutinas preespecificadas y experimentadas por los expertos o científicos". Harlen (1988), comentando este mismo aspecto, señala que cuando los profesores reciben del exterior las instrucciones de todo lo que han de hacer, tema a tema, transfieren la responsabilidad de la enseñanza a los programas y libros de texto.

EI modelo de currículo adoptado por el MEC es un modelo abierto, pero que pretende tener una función orientadora y garantizar la adquisición de unos contenidos educativos básicos, considerados socialmente relevantes para toda la población escolar.

Difiere en este sentido tanto de los currículo totalmente abiertos, propios de la enseñanza primaria de algunos paises europeos, como de los totalmente cerrados, que prescriben hasta el último detalle los objetivos y contenidos educativos y su secuenciación a lo largo de los diferentes niveles educativos (Coll 1989).

La opción tomada establece un reparto de competencias en el que se diferencian tres niveles de concreción en el diseño del currículo:

Un primer nivel de concreción, competencia de la administración educativa, que recibe el nombre de Diseño Curricular Base (documento presentado actualmente a debate), en el que se definen los objetivos generales para cada etapa educativa, y los objetivos gene- rales y contenidos para cada área curricular para toda la etapa.

Un segundo nivel de concreción, competencia de los equipos educativos de centro, que implica la organización y secuenciación de los objetivos y contenidos educativos para los distintos ciclos de cada etapa, y la defenición global de las opciones metodológicas, de los recursos a utilizar y de los criterios de evaluación. Este conjunto de decisiones configura el Proyecto Curricular de Centro.

Un tercer nivel de concreción, competencia de cada profesor, en el que se definen los objetivos y contenidos específicos, junto con las actividades de enseñanza y evaluación, para cada grupo clase de un nivel educativo concreto, Io cual conforma la programación de actividades.

Esta opción no está carente de riesgos, ya que se trata de alcanzar un difícil equilibrio entre el grado de prescripción y el grado de apertura, imposible de satisfacer a todos los sectores sociales y profesionales. Junto a elio hay que señalar también la falta de tradición del modelo, la poca familiarización con el mismo del profesorado y las nuevas capacidades profesionales que requiere su utilización. No obstante estas dificultades no deben servir, como indica Harlen (1989), para seguir coartando la libertad curricular de los profesores, sino más bien para reconocer la dificultad de las decisiones que se tomen, y proporcionar la estructura de apoyo necesaria.

Al lado de los riesgos considerados en este modelo de desarrollo curricular, propios por otra parte de toda opción innovadora, que aborda de forma racional ciertos problemas, deben valorarse también las opciones que abre, de cara a mejorar la calidad de la enseñanza y la profesionalidad docente.

Heathcote y colaboradores (1982) han identificado tres estrategias básicas para el desarrollo curricular:

* Desarrollo del centro (administración educativa) a la periferia (centros).

* Desarrollo curricular en colaboración.

* Innovacion centrada en la escuela.

El modelo adoptado por el MEC en las nuevas propuestas se aproxima a la segunda de estas estrategias.

Estos mismos autores señalan que en los últimos años se observa un desplazamiento del primer modelo (el más prescriptivo y centralizado) hacia los otros dos, sin considerarios en ningún momento excluyentes.

Garret (1988) atribuye este desplazamiento, por una parte, a la necesidad de eliminar barreras entre la innovación curricular y su implementación, y por otra al reconocimiento de que los profesores están, con frecuencia, en mejor posición para identificar los problemas referentes al currículo, y posiblemente para resolverlos. 
Al mismo tiempo es necesario considerar el importante papel formativo de una participación, lo más activa posible, del profesorado en el desarrollo del currículo ya que, tal como señala Stenhouse (1980a), el currículo es el medio a través del cual el profesor puede aprender su arte y la naturaleza de los conocimentos que imparte. Y esto es así porque el desarrollo del currículo le permite poner a prueba ideas por obra de la práctica y, en consecuencia, basarse en su juicio más que en el juicio de los demás.

Por último hay que hacer referencia a la contribución de un currículo abierto al desarrollo profesional. En palabras de Gimeno (1987): "El desarrollo de la profesionalidad de los profesores exige que éstos participen en el diseño de la enseñanza como recurso para ejercer la creatividad de su profesión y un cierto control sobre la misma".

\section{LOS GRANDES PROYECTOS CURRICU. LARES DE CIENCIAS}

El término proyecto curricular tiene ya una larga tradición en el campo de la práctica educativa. Su uso se asocia normalmente a los grandes proyectos curriculares, que fueron objeto de atención preferente por parte de las políticas curriculares de varios países en los años 60. Impuisados por esta polf́tica de desarrollo curricular, y con el soporte de numerosas instituciones públicas y privadas, se produjeron gran cantidad y variedad de proyectos curriculares para la enseñanza de las ciencias y otras materias escolares, en los distintos niveles educativos.

Estos proyectos curriculares, se elaboraban en general con una fuerte dotación de recursos económicos y humanos, y tenían como objetivo básico elaborar conjuntos de materiales estructurados, que ofrecieran al profesor desarrollos curriculares concretos para las disciplinas cientifficas.

Surgieron como una alternativa al libro de texto tradicional y pretendían facilitar una nueva forma de enseñar las ciencias, tanto a nivel metodológico, situándose en general dentro de una concepción amplia de enseñanza activa, como en relación a la forma de organizar y presentar los contenidos educativos.

Las opciones elaboradas fueron muy variadas, ya que se financiaron distintos proyectos simultáneamente, $\mathrm{y}$ la inversión económica realizada fue muy grande, principalmente en Estados Unidos y el Reino Unido.

Su elaboración corría a cargo de equipos interdisciplinares, con un fuerte apoyo investigador y una participación variable del profesorado en ejercicio. Los procesos de experimentación, en general, eran amplios y seguidos de una evaluación sistemática, en base a la que se realizaban reelaboraciones de los proyectos iniciales.

El análisis de estos proyectos rebasa el marco de esta ponencia y existe una amplia bibliografía al respecto
(Caamaño 1988, Pintó 1983, Shayer 1972, 1974, 1978, Stenhouse $1980 \mathrm{~b}$ ). También puede encontrarse un destacado número de trabajos sobre distintos proyectos, en los volúmenes editados por la Unesco, dedicados a revisar las líneas de innovación en la enseñanza de la biología, la física, las matemáticas, la química y la ciencia integrada (New trends in biology teaching, in chemistry teaching in mathematics teaching, in physics teaching, in integrated science teaching).

A nuestro país estos proyectos llegaron tarde (las primeras traducciones se realizaron a principios de los años 70), y tuvieron una repercusión muy limitada en los centros educativos debida, entre otras causas, a la dificultad de adecuarlos a los programas vigentes y a las rígidas estructuras horarias, a la falta de recursos adecuados para su implementación, a Ia ausencia de una formación específica del profesorado para su utili. zación y a la falta de apoyos institucionales. Sin embargo, tuvieron una importancia fundamental para los grupos de innovación, que en muchos casos los adoptaron como material de referencia y que, con recursos y apoyo, realizaron adaptaciones de algunos de ellos, liegando en algunos casos a crear sus propios proyectos experimentándolos en un importante número de centros, si se tienen en cuenta las precarias condiciones en las que se movían.

Estos proyectos tienen el gran valor de haber aportado una visión científica y psicológica del tratamiento de las ciencias, que contrasta con la pobreza de los enfoques de los libros de texto más tradicionales. Al mismo tiempo ofrecen bancos de actividades experimentales, recursos, orientaciones didácticas y para la evaluación al profesorado. Por otra parte el movimiento de reforma del currículo de ciencias de los años 60 dio un importantísimo impulso a la investigación didáctica, generando modelos de diseño, experimentación y evaluación de gran validez.

En varias de las evaluaciones realizadas de estos proyectos, se constata con frecuencia una escasa incidencia en la mejora del aprendizaje de las ciencias, en ocasiones atribuida a la inadecuación de su nivel a las capacidades cognitivas de los alumnos (Shayer 1972, $1974,1978,1984$ ), y en otras, a la falta de formación del profesorado (Ausubel 1983, Elliot 1985). Ello, ha Ilevado en los últimos años a un progresivo abandono de la política de grandes proyectos curriculares, atribuible, con todas las matizaciones necesarias, a razones económicas y a la poca optimización de los resultados conseguidos en función de las importantes inversiones y recursos movilizados.

Garret (1988) señala una tendencia general al abandono de los grandes proyectos curriculares en favor de intentos más modestos, y comenta también que los grandes grupos de trabajo están cediendo su lugar a proyectos locales basados en la participación de los profesores. Atribuye esta tendencia por una parte a que, si bien los grandes proyectos curriculares, al estar elaborados por expertos, tenian una notable calidad científica, no contemplaban elementos importantes del contexto en que éstos debían aplicarse. Por otra parte, 
con frecuencia el profesor recibía estos materiales sin ir acompañados del proceso formativo necesario. Ello conducía a una utilización inadecuada de las propuestas, no consiguiéndose los objetivos planteados en el proyecto original.

Sin infravalorar las importantes aportaciones de los grandes proyectos curriculares, y considerando que eI desarrollo de nuevas estrategias curriculares ha sido posible gracias al movimiento de reforma del currículo de ciencias de los años 60 , se justifica a continuación la concepción del desarrollo curricular propugnada en la propuesta de Diseño Curricular Base elaborada por el MEC.

\section{LOS PROYECTOS CURRICULARES DE CENTRO}

En la propuesta de Diseño Curricular Base se diferencia el Proyecto Curricular de Centro, conjunto de decisiones articuladas, tomadas por los equipos docentes, que suponen la concrección del DCB en propuestas de intervención didáctica adecuadas a un contexto específico, de los materiales curriculares, conjunto de recursos elaborados externamente a la escuela, que los equipos docentes pueden utilizar parcial o globalmente para elaborar sus proyectos propios.

Esta diferenciación representa una reconceptualización del uso clásico del término proyecto curricular, en el sentido de que los grandes proyectos curriculares elaborados por equipos externos a la escuela, pasan a ser considerados materiales de desarrollo curricular, reservándose el uso đel término proyecto curricular para los desarrollos curriculares contextualizados en los centros. Esto no implica una infravaloración de los materiales curriculares, sino resituar su función dentro de una concepción distinta del desarrollo curricular, en la que se atribuye un nuevo papel al profesorado.

Esta reconceptualización ha sido criticada por algunos autores (Gimeno 1989), que la consideran confusa y poco realista. Evidentemente en la propuesta realizada por el MEC, no se plantea que los equipos de centro elaboren proyectos al estilo de los grandes proyectos curriculares de los años 60 , como Gimeno interpreta; pretensión que resultaría como mínima ingenua. Tal como señala Harlen (1989) no es razonable esperar que los profesores hagan por su cuenta lo que diversos planificadores del currículo han estado desarrollando durante muchos años.

El importante papel atribuido a los equipos docentes en la elaboración de los proyectos curriculares de centro deriva de la consideración de que la mayor funcionalidad de estos requiere que sean asumidos colectivamente, $y$ que haya un grado elevado de reflexión y comprensión de los mismos. Y se considera que la mejor forma de garantizar esto es mediante una elaboración colectiva, realizada en el propio contex to de aplicación. Ello no implica que un proyecto curricular de centro no pueda asumir de forma parcial o global propuestas elaboradas fuera de la escuela, pero esta opción deberá ser precedida de un trabajo en equipo de reflexion y de análisis y de una adecuación específica a las condiciones del centro.

Los materiales de desarrollo curricular elaborados externamente a los centros pueden y deben ser una ayuda fundamental para los equipos docentes, pero no deben suplir su propia toma de decisiones; ya que, tal como comenta Ángel Pérez (1986): "Cuando se prespecifican excesivamente las categorías de intervención en el marco curricular, o cuando los proyectos curriculares se adoptan no como ejemplos sino como modelos únicos e ideales de intervención, se invita al profesor a renunciar a su propia actuación como artista, se mutila su espacio de creación y se cercenan las posibilidades de encuentro entre el conocimiento científico y el conocimiento empírico del alumno".

Estas afirmaciones no derivan únicamente de supuestos teóricos, sino simultáneamente del análisis de las experiencias de innovación realizadas en los centros educativos (del Carmen Mauri, Solé y Zabala 1988). Si se reflexiona sobre ellas, puede constatarse fácilmente que en aquellos centros donde se han realizado proyectos innovadores de calidad se han dado con frecuencia dos condiciones: un trabajo en equipo de reflexión y fundamentación sobre la práctica educativa, y una actividad creativa de adaptación de propuestas externas ya elaboradas o de elaboración de propuestas propias, adecuadas al contexto específico del centro. La aplicación mecánica de proyectos externos, sin un marco de reflexión y análisis colectivo, orientado a elaborar respuestas adaptadas a las condiciones específicas, difícilmente produce efectos innovadores y de mejora en la calidad de la enseñanza. Y es esta limitación la que intenta superarse.

La reconceptualización planteada, por tanto, no es de carácter formal, sino que afecta a la manera de entender el desarrollo curricular. En este sentido es totalmente válida si se considera que la reconceptualización es un procedimiento científico de uso generalizado, que puede posibilitar el progreso tanto teórico como práctico.

Por otra parte esta forma de entender el desarrollo curricular es coherente con una concepción constructivista del aprendizaje y la intervención pedagógica, para la que es fundamental contemplar los conocimientos previos de los alumnos y adecuar los procesos de enseñanza a sus necesidades y contex tos específicos, lo cual no puede ser realizado de forma concreta sin conocerlos.

Ahora bien, es cierto, tal como Gimeno (1987) señala, que: "En una situación concreta, para la renovación educativa han de ofertarse esquemas que sean útiles al profesorado desde el estado de sus propias necesidades para progresar hacia una mayor autonomía profesional". En este sentido será necesario contemplar y garantizar la elaboración de una gama variada de materiales de desarrollo curricular, que permitan la incorporación progresiva del profesorado, partiendo de su situación y necesidades actuales, pero potenciando al mismo tiempo su autonomía en la reflexión y la toma de decisiones. 
Por todo ello, para que el desarrollo curricular pueda basarse en un modelo participativo como el que se plantea, será necesario abocar esfuerzos por parte de todos los sectores implicados. Estos esfuerzos deben estar orientados a crear una organización en los centros que lo posibilite, y a proporcionar la orientación y ayuda formativa externa que los equipos necesiten. En palabras de Harlen (1989): “...debe preservarse la máxima libertad del profesor, proporcionándole al mismo tiempo la mayor ayuda posible para que la emplee eficazmente".

Como factores externos a los centros para favorecer este proceso, se consideran fundamentales:

* El establecimiento de unas condiciones para el ejercicio de la profesión docente, coherentes con estos planteamientos.

* La realización de planes generales de formación permanente, dirigidos a los equipos docentes de los centros, que les ayuden a desarrollar una dinámica propia de elaboración de sus proyectos.

* La elaboración, por encargo de las administraciones educativas, de materiales orientadores para el tratamiento de las áreas curriculares (el informe Cockroft para la enseñanza de las matemáticas en el Reino Unido puede servir de ejemplo).

* La elaboración y difusión de materiales de desarrollo curricular, coherentes con los planteamientos del Diseño Curricular Base, que ayuden a los equipos docentes a progresar en la elaboración de los proyectos curriculares de centro.

* Y el asesoramiento efectivo y continuado a los equipos de centro que lo soliciten.

Aun teniendo en cuenta que este conjunto de medidas sólo pueden ponerse en funcionamiento de forma progresiva, y a partir de los recursos económicos y humanos existentes, debe considerarse que su implementación determinará en gran medida el grado de éxito del tipo de desarrollo curricular planteado, y que las deficiencias en las mismas facilitarán el retorno a la solución más sencilla del currículo cerrado (por las propias administraciones o por las editoriales).

\section{ELEMENTOS BÁSICOS DEL PROYECTO CURRICULAR DE CENTRO}

E1 Proyecto Curricular de Centro se ha definido (Del Carmen y Zabala 1988) como el conjunto de decisiones articuladas tomadas por los equipos docentes de los centros escolares, tendentes a dotar de mayor coherencia su actuación, con la finalidad de concretar el Diseño Curricular Base en propuestas globales de inter. vención didactica, adecuadas a un contexto específico. Para poder definir los elementos básicos que deben configurar un proyecto curricular de centro, es necesario precisar qué necesidades debe cubrir. Si se tiene en cuenta que el Diseño Curricular Base define los objeti- vos generales de cada etapa, y los objetivos generales y contenidos de cada área para la etapa correspondiente, el proyecto curricular de centro deberá precisar todos los elementos que suponen una decisión del equipo de profesores de etapa, de una o más áreas, y que son previos a la elaboración de las programaciones de actividades para un nivel educativo determinado.

La elaboración del PCC requiere tomar las siguientes decisiones básicas:

* Adecuar los objetivos generales del área planteados en el DCB a las características específicas del centro y precisar los objetivos generales para cada ciclo.

* Analizar, secuenciar y organizar el conjunto de contenidos educativos que serán desarrollados.

* Establecer los objetivos terminales de etapa y ciclo.

* Definir y temporalizar los temas básicos a trabajar en cada ciclo, y las opciones metodológicas que deben orientarlos.

* Establecer los criterios para la selección de materiales curriculares y otros recursos didácticos.

* Definir los criterios de organización espacio-temporal.

* Precisar los criterios de evaluación interciclos.

Todas estas cuestiones son de especial importancia si se tiene en cuenta que, tanto las ciencias experimentales, como las matemáticas, forman parte de áreas curriculares que se imparten durante todos los cursos, tanto en la Educación Primaria, como en la Secundaria obli. gatoria. La elaboración del PCC tiene como objetivo fundamental garantizar una actuación coherente, coordinada y progresiva a lo largo de la escolaridad obliga* toria, que favorezca al máximo el desarrollo global y diversificado de los alumnos. Esto requiere que las distintas actuaciones educativas que inciden sobre ellos vayan orientadas en la misma dirección, para garantizar su eficacia y el equilibrio y crecimiento personal de los alumnos.

La elaboración del proyecto curricular de centro implica, como señaia Harlen (1989), menor libertad para cada profesor individual, pero no supone una disminución de la libertad para el claustro de profesores como un todo, si se compromete a poner en práctica las decisiones aceptadas para el conjunto del centro.

De todos los elementos señalados como fundamentales para la elaboración de un proyecto curricular de centro, vamos a centrarnos en el análisis de uno de ellos, por considerar que representa una mayor novedad en las competencias de los equipos docentes, y que por otra parte tiene un mayor grado de dificultad: es el relativo a la secuenciación de los contenidos objeto de enseñanza.

\section{LA SECUENCYACIÓN DE LOS CONTENIDOS EDUCATIVOS}

En el DCB los contenidos se presentan para toda la etapa, por io que se hace necesario establecer a nivel de 
centro una secuenciación de los mismos, que proporcione una progresión adecuada que favorezca los aprendizajes.

El tema de la secuenciación de contenidos ha sido poco desarrollado en comparación con otros temas curriculares. Uno de los primeros autores en estudiar de forma amplia y sistemática el problema de la secuenciación ha sido Gagné (1965). Para él la condición fundamentail para establecer secuencias de instrucción adecuadas radica en respetar las jerarquías de aprendizaje. Éstas nos muestran las relaciones de requisito que hay en el desarrollo de los objetivos educativos.

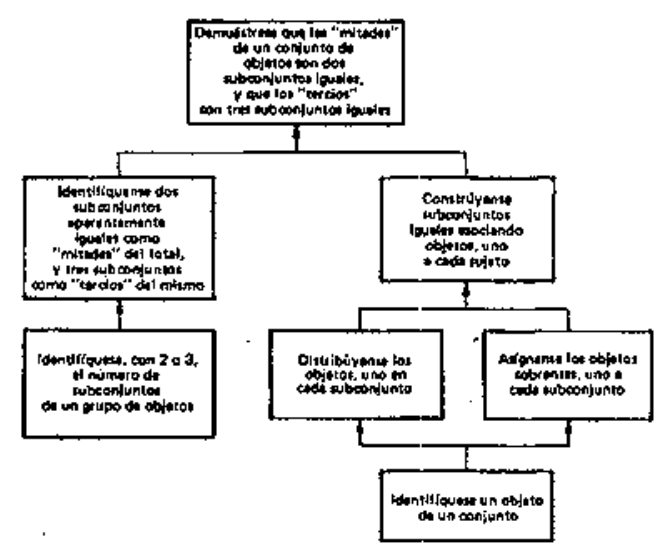

Jerarquía de aprendizaje de una tarea de matemáticas elemental. (Tomado de Gagné, R. M., 1970; figura 17, adaptada de Resnick y Wang, 1969.)

Para establecer las jerarquías de aprendizaje Gagné se basa en el análisis de tareas (Gagné y Brigs 1974, Brigs 1973, Araujo y Chadwick 1988, Gutiérrez 1989). Para aprender a realizar correctamente una tarea determinada, el alumno debe haber adquirido unas habilidades previas. Éstas a su vez se apoyarán en otras habilidades ya adquiridas. Las habilidades de requisito se consideran esenciales para el aprendizaje de la habilidad definiđa en el objetivo con que se inició la jerarquía; cada una de ellas tiene sus propios requisitos, que se indican en el siguiente nivel de la jerarquía. De esta forma la tarea inicial, definida en base a la conducta terminal esperada, se descompone en objetivos de ejecución cada vez más simple, que deben ser dominados previamente.

La limitación más importante de las consideraciones de Gagné, es que, si bien el análisis de tareas que plantea en términos de los requisitos de aprendizaje necesarios para realizarlas proporciona una gran información sobre la naturaleza y complejidad de las mismas, nos dice muy poco sobre como pueden llegar a dominarse, ya que como indica Pozo (1987), "...la estructura total de conocimientos no es simplemente una suma de las partes que la componen, sino que posee una dinámica propia".

Por otra parte, tal como señala Coll (1987), hay contenidos de la enseñanza que resultan difíciles de traducir en objetivos de ejecucion. Además el establecimiento de jerarquías de aprendizaje es más fácil en relación a capacidades simples, pero cuando éstas son complejas resultan muy difíciles de establecer. Por ello la secuenciación basada únicamente en las jerarquías de aprendizaje corre un doble riesgo: dejar de lado aspectos educativos importantes, para los que no puedan encontrarse correlatos de ejecución y desatender intenciones educativas relativas a aprendizajes complejos.

Otro enfoque para secuenciar los contenidos educativos deriva de la teoría de los estadios de Piaget (1936, 1955). Aunque Piaget no formuló directamente prescripciones en relación a la forma de organizar y secuenciar los contenidos educativos, algunos autores (Shayer y Adey 1984), apoyándose en la teoría de los estadios, han establecido taxonomías que permiten analizar el grado đe dificultad de los contenidos, en función de las operaciones lógicas que éstos implican y secuenciarlos en función del mismo.

Este análisis, si bien permite detectar el grado de dificultad de los contenidos educativos en relación al nivel de desarrollo operatorio de los alumnos, es poco orientativo de cara a establecer secuencias de instrucción adecuadas, ya que nos indica lo que un alumno puede hacer en un momento determinado, pero no lo que es capaz de aprender. Por otra parte las tareas razonadas, utilizadas para detectar el nivel operatorio de los alumnos, presupone la transferencia de las capacidades operatorias a cualquier tipo de contenidos, aspecto de la teoría de Piaget que ha sido fuertemente cuestionado (Vuik 1984).

La teoría del aprendizaje significativo de Ausubel (1968), aborda el problema de la organización y secuenciación de los contenidos curriculares centrándose en los componentes de tipo conceptual. Para este autor la organización cognitiva del individuo está estructurada conceptualmente y los conceptos actúan como mediadores entre el individuo y el medio. La adquisición de nuevos conocimientos está por tanto básicamente determinada por las estructuras conceptuales previas del individuo.

Ausubel considera que las habilidades intelectuales y los aspectos afectivos y sociales influyen en los aprendizajes, pero los considera poco útiles para organizar la enseñanza escolar, por tanto las prescripciones de su teoría se centran en los aspectos conceptuales de las disciplinas.

El aprendizaje significativo se produce a través de una interacción de la nueva información con las ideas pertinentes que existen en la estructura cognoscitiva del que aprende (inclusores). Como resultado de esta interacción se produce una asimilación de significados, que da lugar a una estructura cognoscitiva más diferenciada. Esta estructura cognoscitiva tiene una organización jerárquica, que es necesario respetar para facilitar el aprendizaje significativo.

Durante el curso del aprendizaje significativo se distinguen dos procesos de gran importancia: la diferenciación progresiva y la reconciliación integradora. A medida que se producen nuevos aprendizajes, los conceptos 
inclusores se modifican y desarrollan, haciéndose cada vez más diferenciados (diferenciación progresiva). Este proceso produce una estructura cognoscitiva organizada jerárquicamente, cada vez más precisa y con mayo. res posibilidades de aprendizaje significativo, ya que cada vez aumentan las ideas relevantes en las que pueden anclarse nuevos conceptos. Por otra parte la reconciliación integradora permite el establecimiento de nuevas relaciones entre conceptos.

Cuando vamos a estudiar un nuevo grupo de animales, que el alumno no conoce como tal (p. ej.: los crustáceos), podemos utilizar el concepto más general de animal que el alumno ya posee, aunque en un nivel poco diferenciado, como concepto inclusor, de forma que pueda reconocer en el nuevo grupo las características generales ya conocidas de los animales. Esto hace que su concepto de animal se enriquezca y diferencie más (diferenciación progresiva). Al mismo tiempo resulta importante ilustrar el nuevo concepto adquirido con ejemplos ya conocidos por el alumno (cangrejos, gambas, cigalas), y compararlo con otros grupos de animales ya estudiados (moluscos, gusanos), para que afiance sus características propias (reconciliación integradora).

A partir de estas premisas básicas, al establecer las secuencias de enseñanza se recomienda presentar primero las ideas básicas de la disciplina, antes de introducir los conceptos más periféricos. La diferenciación progresiva de los contenidos facilitará el paso de lo más general e inclusivo a lo más detallado y específico. Al mismo tiempo deben establecerse relaciones entre contenidos del mismo nivel para facilitar la reconciliación integradora.

La propuesta de abordar la secuenciación de contenidos estableciendo jerarquías conceptuales es, tal como señala Coll (1987), compatible con una interpretación constructivista del aprendizaje escolar y de la enseñan$z a$, ya que tiene en cuenta simultáneamente la estructura interna de los contenidos y los procesos psicológicos de los alumnos. Sin embargo, presenta el grave inconveniente de centrarse de forma exclusiva en los componentes conceptuales. Para superar esta limitación es necesario dar cabida en los criterios de secuenciación a otros tipos de contenidos y aplicar a ellos los principios del aprendizaje significativo. En esta línea se sitúa la teoría de la elaboración (Reigeluth y Stein 1983).

La teoría de la elaboración tiene como propósito fundamental prescribir criterios para seleccionar, secuenciar y organizar los contenidos educativos, de forma que se consiga una optima adquisición, retención y transferencia de los mismos.

Es una teoría que integra diferentes aportaciones. De Gagné adopta la noción de prerrequisitos de aprendizaje, en el sentido de aceptar que existen determinados conocimientos que deben ser adquiridos antes que otros. De Bruner toma la noción de currículo en espiral, que postula la necesidad de organizar la instrucción en torno a unas ideas eje, que son retomadas sucesivamente en Ios distintos niveles educativos, con un grado progresivo de profundizacion. De las recientes aporta. ciones de la psicología cognitiva, recoge el concepto de esquema de conocimiento y la importancia de prestar una atención explícita a los componentes metacognitivos (estrategias de conocimiento). Pero sin duda la influencia más importante la recibe de las aportaciones de Ausubel.

La teoría de la elaboración considera que los procesos de enseñanza deben comenzar proporcionando una visión de conjunto de los contenidos que van a ser enseñados (siempre que éstos estén fuertemente rela cionados). En esta visión de conjunto deben presentarse las ideas más generales, simples y fundamentales, pasando después a elaborar cada una de ellas y regresando periódicamente a la visión de conjunto, con el fin de ampliarla y enriquecerla. Esto no quiere decir que todos los elementos de contenido presentes en los primeros niveles tengan que ser retomados con más detalle en los niveles superiores. Tampoco que en el primer nivel se haya de presentar un resumen de todos los contenidos, sino una selección de los más fundamentales, que proporcionen una base con la que rela* cionar los demás.

Por ejemplo, el estudio de los seres vivos debería ini. ciarse con una presentación de las características comunes a todos ellos, pero de forma simple y concreta. En este sentido sería mejor empezar por una presentación de las características básicas de los seres vivos, reconocibles y familiares a los alumnos (nacimiento, alimentación, crecimiento, reproducción, muerte), para pasar en un primer nivel de elaboración a analizar cada una de estas características con mayor profundidad, y volver al final de la secuencia a sintetizar las caracte. rísticas básicas de los seres vivos, con mayor grado de precisión, complejidad y abstracción. No se consideraría sin embargo adecuado, desde la perspectiva de la teoría de la elaboración, comenzar presentando como característica común de los seres vivos el estar forma" dos por células o por un tipo especial de moléculas, si los alumnos no poseen aún referentes propios, que les permitan identificar esto como una característica común de todos los seres vivos.

El epítome es el elemento que permite articular las secuencias de enseñanza. En él se presentan un pequeño número de ideas generales y simples, en un nivel concreto de aplicación. El epítome es objeto de enseñanza (constituye la primera unidad de la secuencia), y sus elementos se eligen de forma que el resto de contentidos que se desarrollen a continuación aporten detalles o complejidad.

El proceso de elaboración del epítome requiere:

* Seleccionar un tipo de contenido organizador, que pueden ser: conceptos, principios o procedimientos.

* Analizar el contenido elegido.

* Analizar los prerrequisitos de aprendizaje.

* Seleccionar los elementos más representativos y fundamentales de contenido, que serán presentados en el epítome. 
* Seleccionar los contenidos de soporte (contenidos auxiliares necesarios para el desarrollo de los contenidos fundamentales).

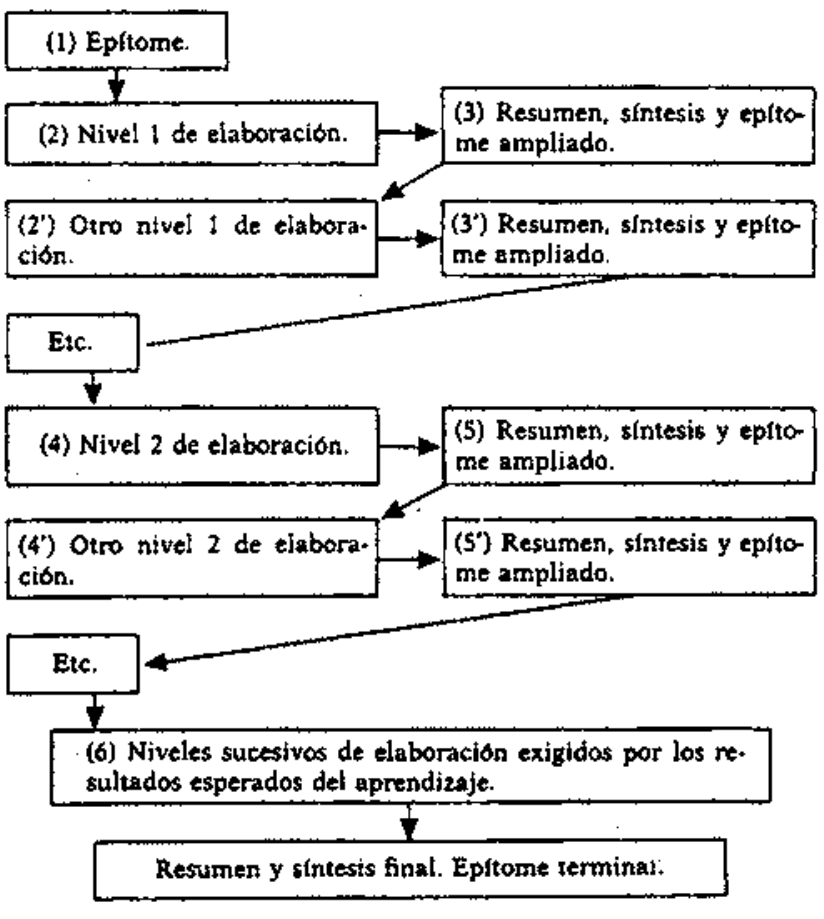

Esquema del proceso de elaboración de las secuencias de instrucción (Adaptado de Reigeluth y Stein, 1983).

La profundidad a la que se sitúa el nivel de elaboración está condicionada por los conocimientos previos del alumno. Cada nivel de elaboración acaba con un resumen, una síntesis y un epítome ampliado, que puede ser el punto de partida para iniciar otra secuencia de unidades. El epítome ampliado es el epítome inicial enriquecido a partir del análisis realizado y las nuevas relaciones establecidas.

Este proceso puede repetirse tantas veces como se considere oportuno a lo largo del currículo, hasta lograr el grado de profundidad deseado.

La teoría de la elaboración aporta un conjunto de criterios articulados y coherentes, de gran utilidad para establecer secuencias de contenidos, ya que:

* Ayuda a la formación de estructuras cognitivas más estables (mayor retención a largo plazo y mayor posi- bilidad de transferencia), facilitando la interrelación entre los procesos de análisis y síntesis.

* Ayuda a la creación de contextos significativos en relación a los contenidos instruccionales y provoca por tanto mayor motivación en los alumnos.

* Proporciona un conocimiento general sobre los aspectos principales del contenido, permitiendo un mayor control, tanto del profesor como de los alumnos sobre la selección y secuenciación de contenidos.

Aunque la teoría de la elaboración tiene en cuenta además de los contenidos conceptuales (conceptos y principios) los procedimentales, no hace referencia a los de tipo actitudinal. Teniendo en cuenta que entre los contenidos de la educación obligatoria, los relacionados con las actitudes, valores y normas tienen una gran importancia, deberian encontrarse criterios de tratamiento adecuados para evitar que queden relegados en el proceso de enseñanza.

En el Diseño Curricular Base en cada bloque de contenidos, junto a los conceptuales y procedimentales, se especifican contenidos actitudinales; sin embargo no existen aún modelos suficientemente elaborados, que permitan integrar en la planificación de los procesos de ensefranza los distintos tipos de contenidos.

Giordan (1978), a partir de estudios empíricos, establece unas secuencias, que permiten orientar la observación del desarrollo de las actítudes científicas generales en los alumnos. Aunque las pautas aportadas son de gran utilidad, resultan demasiado generaies para orientar el establecimiento de secuencias específicas de enseñanza.

En la medida que las investigaciones sobre el desarrollo de actitudes científicas en los alumnos están siendo, cada vez más, objeto de atención, son de esperar futuras aportaciones que integren de una forma más efectiva los distintos tipos de contenidos en un modelo global de enseñanza/aprendizaje.

Abordar los problemas presentados supone un importante reto para los equipos docentes, tanto por la poca formación recibida en relación a ellos, como por su complejidad. Sin embargo es de esperar que las administraciones educativas, conscientes de esto, proporcionen la máxima ayuda en relación a la formación permanente, la elaboración de materiales orientativos y el desarrollo de las investigaciones pertinentes. En cualquier caso el análisis y discusión de toda esta problemática contribuirá a un enriquecimiento del profesorado y a ampliar las perspectivas, tanto teóricas como prácticas, de la profesión docente. 


\section{REFERENCIAS BIBLIOGRÁFICAS}

ARAUJO, J.B. y CHADWICK, C.B., 1988. Tecnología educacional: teorias de la instrucción. (Paidós: Barcelona).

AUSUBEL, D.P., 1976. Psicología Educativa: un punto de vista cognoscitivo. (Trillas: México). (Ed. Orig: 1968, Educational Psychology: A cognitive view. (Holt, Rinehart \& Winston: New York).

AUSUBEL, D.P., NOVAK, J.D. y HANESIAN, H., 1983. Psicología educativa: un punto de vista cognoscitivo. (Trilias: México)

BRIGS, L.J., 1973. El ordenamiento de la secuencia en la instrucción. (Guadalupe: Buenos Aires).

CAAMAÑO, A., 1988. Tendencias actuales en el currículo de ciencias, Enseñanza de las Ciencias, 6(3) pp. 265-277.

COLL, C., 1987. Psicología y currículum. (Laia: Barcelona).

COLL, C., 1989. Diseño Curricular Base y Proyectos Curriculares, Cuadernos de Pedagogía, 168, pp. 8-14.

DEL CARMEN, L., MAURI, T., SOLÉ, I, y ZABALA, T., 1988. Innovación pedagógica, diseño curriculary desarrollo del currículum. Ponencia presentada en las Jornadas de directores de Centros de Profesores, Subdirección de Formación del Profesorado, Madrid.

DEL CARMEN, L. y ZABALA, T., 1988. Orientaciones y criterios para la elaboración de Proyectos Curriculares de Centro. Documento inédito, Departamento de Psicología Evolutiva y de la Educación, Universidad de Barcelona.

ELLIOT, \}., 1985. El profesor como investigador. Ponencia presentada en las $/ I I$ Jornadas de Investigación en la Escuela, Sevilla.

GAGNÉ, R.M., 1971. Las condiciones del aprendizaje. (Aguilar: Madrid). (Ed. Orig: 1965, The conditions of learning. (Holt, Rinehart \& Winston: New York).

GAGNE, R.M. y BRIGS, L.J., 1976. La planificación de la enseñanza. (Trillas: México).

GARRET, R.M., 1988. Adaptacion curricular "del fin hacia el principio": una táctica alternativa para el desarrollo curricular, Investigación en la Escuela, 5, pp. 3-10.

GIMENO, J., 1987. Las reformas curriculares y el profesorado, en Amelia Álvarez (Comp.), Psicología y educación. Actas de las $1 /$ Jornadas Internacionales de Psicología y Educación. (Visor/Aprendizaje: Madrid).

GIMENO, J., 1989. Proyectos curriculares. ¿Posibilidad al aicance de los profesores?, Cuadernos de Pedagogía, 172, pp. 14-18.

GIORDAN, A., 1982. La enseñanza de las ciencias. (Siglo XXI: Madrid). (Ed. Orig: 1978, Une pédagogie pour les ciences expérimentales. (Centurion: París).
GUTIÉRREZ, R., 1989. Psicología y aprendizaje de las Ciencias. EI modelo de Gagné, Enseñanza de las Ciencias, 7(2), pp. 147-157.

HARLEN, W., 1989. Enseñanza y aprendizaje de las ciencias. (Morata: Madrid).

HEATHCOTE, G., KEMPA, R. y ROBERTS, 1., 1982, $\mathrm{Cu}$ rriculum Styles and Strategies, a project report for the Further Education Curriculum Review and Development Unit. (DES Publications Despatch Center: Middlesex).

MEC, 1989. Diseño Curricular Base. (MEC: Madrid).

PÉREZ, A., 1986. Más sobre la formación del profesorado, Cuadernos de Pedagogía, 139, pp. 92-94.

PIAGET, J., 1969. El nacimiento de la inteligencia en el niño. (Aguilar: Madrid), (Ed. Orig: 1936, La naissance de l'intelligence chez l'enfant. Delachaux \& Niestlè: Neuchâtel, París).

PLAGET, J. y INHELDER, B., 1972. De la lógica del niño a la lógica del adolescente. (Paidós: Buenos Aires). (Ed. Orig: 1955, De la logique de l'enfant à la logique de l'adolescent. Presse Universitaires de France: París).

PINTÓ, R., 1983. Andisi de programes de Física per a l'ensenyament elemental. (Universitat Autònoma de Barcelona: Bellaterra).

POZO, I., 1987. Aprendizaje de la ciencia y pensamiento causal. (Aprendizaje/Visor: Madrid).

REIGELUTH, CH.M. y STEIN, F.S., 1983. The elaboration Theory of instruction, en Ch. M. Reigeluth (ed.), Instructional Design Theories and Models : an overview of their current status. (Hillsdale: Erlbaum).

SHAYER, M., 1972. Conceptual demands in the Nuffield Olevel Physics, School Science Review 186, pp. 26-34.

SHAYER, M., 1974. Conceptual demands in the Nuffield Olevel Biology Course, School Science Review, 56, pp. 381 388.

SHAYER, M., 1978. Nuffield Combined Science: do the pupils understand it?, School Science Review, 60, pp. $210-$ 223 .

SHAYER, M. y ADEY, P., 1984. La ciencia de enseñar Ciencias (Narcea: Madrid).

STENHOUSE, L., 1980a, Curriculum research and the art of the teacher, reproducido en La investigación como base de la enseñanza (Morata: Madrid).

STENHOUSE, L. (ed.), 1980b. Curriculum Research and Development in Action. (Heinemann Educational Books: London).

VUYK, R., 1984. Panorámica y crítica de la epistemología genética de Piaget. (Alianza: Madrid). 